\title{
openheart Coronary artery calcium, HIV and inflammation in Uganda compared with the USA
}

\author{
Ben Alencherry, ${ }^{1}$ Geoffrey Erem, ${ }^{2,3}$ Grace Mirembe, ${ }^{4}$ Isaac Ssinabulya, ${ }^{3,5}$ \\ Chun-Ho Yun, ${ }^{6}$ Chung-Lieh Hung, ${ }^{7}$ Mark J Siedner, ${ }^{8,9}$ Marcio Bittencourt, ${ }^{10}$ \\ Cissy Kityo, ${ }^{4}$ Grace A McComsey, ${ }^{1,11}$ Chris T Longenecker ${ }^{\oplus 12}$
}

\begin{abstract}
- Additional material is published online only. To view please visit the journal online (http://dx.doi.org/10.1136/ openhrt-2019-001046).
\end{abstract}

To cite: Alencherry B, Erem G, Mirembe G, et al. Coronary artery calcium, HIV and inflammation in Uganda compared with the USA. Open Heart 2019;6:e001046. doi:10.1136/

openhrt-2019-001046

Received 7 March 2019 Revised 11 April 2019 Accepted 26 April 2019
Check for updates

(c) Author(s) (or their employer(s)) 2019. Re-use permitted under CC BY. Published by BMJ.

For numbered affiliations see end of article.

Correspondence to Dr Chris T Longenecker; cx1473@case.edu

\section{ABSTRACT}

Objectives To compare the prevalence of detectable coronary artery calcium (CAC) among higher risk, older people living with HIV (PLWH) and uninfected persons in Uganda versus the USA, and second to explore associations of CAC with HIV-specific variables and biomarkers of inflammation.

Methods This cross-sectional study of 430 total subjects compared 100 PLWH on antiretroviral therapy and 100 age-matched and sex-matched HIV-uninfected controls in Uganda with 167 PLWH on antiretroviral therapy and 63 uninfected controls in the USA. Multivariable logistic regression was used to examine associations with detectable CAC $($ CAC $>0)$.

Results Compared with US subjects, Ugandans were older (mean age 56 vs 52 years) and were more likely to have diabetes ( $36 \%$ vs $3 \%$ ) and hypertension ( $85 \%$ vs $36 \%$ ), but were less likely to be male ( $38 \%$ vs $74 \%$ ) or smokers (4\% vs 56\%). After adjustment for HIV serostatus, age, sex and traditional risk factors, Ugandans had substantially lower odds of CAC $>0$ (adjusted OR 0.07 (95\% $\mathrm{Cl} 0.03$ to 0.17$), \mathrm{p}<0.001)$. HIV was not associated with $C A C>0$ in either country ( $p>0.1)$. Among all PLWH, nadir CD4 count was associated with the presence of $\mathrm{CAC}$, and among Ugandans soluble intercellular adhesion molecule $(p=0.044)$, soluble CD163 $(p=0.004)$ and oxidised low-density lipoprotein $(p=0.043$ ) were all associated with the presence of CAC.

Conclusions Ugandans had a dramatically lower prevalence of any coronary calcification compared with US subjects. The role of HIV infection and inflammation as risk factors for subclinical coronary disease in sub-Saharan Africa merits further investigation.

\section{INTRODUCTION}

In many parts of sub-Saharan Africa (SSA), the prevalence of cardiovascular disease (CVD) appears to be increasing due to rising rates of traditional atherosclerotic cardiovascular disease (ASCVD) risk factors such as obesity, diabetes and hypertension ${ }^{1}$; however, non-traditional risk factors such as chronic infections and exposure to indoor air pollution may also play a role. For example, chronic HIV infection has been associated with a

\section{Key questions}

What is already known about this subject?

- Ischaemic heart disease may be on the rise in sub-Saharan Africa due to recent increases in the prevalence of traditional risk factors and non-traditional risk factors such as HIV infection.

- In addition, modelling analyses using Global Burden of Disease (GBD) Study data suggest that HIV may contribute up to $10 \%-15 \%$ of the population attributable risk for atherosclerotic cardiovascular disease events in sub-Saharan Africa; however, these studies rely on assumptions about risk and disease burden that incorporate very few primary data from the region.

What does this study add?

- This study suggests that despite high rates of risk factors, the prevalence of detectable coronary calcium is much lower among Ugandans compared with US subjects.

- One implication of these findings is that the risk of atherosclerotic coronary heart disease events may be lower in Uganda than predicted by the GBD.

- Finally, our findings suggest that inflammation and immune activation may be important risk factors for coronary artery disease in sub-Saharan Africa, particularly for people living with HIV.

How might this impact on clinical practice?

- These estimates of subclinical cardiovascular disease burden will inform future research on clinical outcomes and may inform health policymakers in low-income countries concerned about controlling non-communicable diseases.

- Our findings should prompt additional investigation of the role of traditional and non-traditional risk factors (such as HIV and inflammation) in the development of coronary disease and clinical cardiovascular events in sub-Saharan Africa.

twofold higher risk of ASCVD and is highly prevalent in SSA. ${ }^{2}$ Modelling studies suggest that HIV may contribute up to $10 \%-15 \%$ of the population attributable risk for ASCVD events in the region. ${ }^{2}$ Yet, although risk 
factors point to increasing risk, little is known about the true prevalence of subclinical vascular disease or clinical ASCVD risk among people living with HIV (PLWH) in SSA. $^{3}$ Some studies of carotid intima-media thickness ${ }^{4}$ and various measures of arterial stiffness ${ }^{5-7}$ have come to mixed conclusions on the relationship between HIV and subclinical vascular disease in varied SSA populations. Yet no prior studies have directly measured the prevalence of subclinical coronary disease among PLWH and controls in SSA using coronary artery calcium (CAC) scores.

The inflammatory milieu of chronic HIV infection that persists despite antiretroviral therapy (ART) - reflected by increased biomarkers of endothelial and monocyte activation in peripheral blood-may explain the higher ASCVD risk among PLWH. ${ }^{8}$ Studies of inflammation biomarkers and subclinical vascular disease among PLWH have, however, been mixed, ${ }^{9}$ with markers of monocyte activation (eg, soluble CD163 (sCD163) and soluble CD14 (sCD14)) showing the most consistent positive associations with the disease. ${ }^{10-12}$ In rural south-west Uganda, higher sCD14 and interleukin-6 (IL-6) levels 6 months after ART initiation appear to be associated with higher carotid intima-media thickness years later. ${ }^{13}$ However, few other studies in the region have examined the association between inflammation and subclinical vascular disease among PLWH in SSA, and none have done so using CAC as a measure of coronary atherosclerosis.

Therefore, we sought to describe the prevalence of detectable CAC among higher risk, older Ugandans with and without HIV in comparison with persons with and without HIV in Cleveland, Ohio, USA. As a secondary objective, we explored associations between calcified coronary plaque and HIV-specific factors in Uganda, including biomarkers of inflammation and immune activation.

\section{METHODS}

\section{Participant selection}

From April 2015 to May 2017, 100 PLWH on ART over 45 years of age and in care at Joint Clinical Research Centre HIV clinic near Kampala were enrolled in the study. For each PLWH, we prospectively identified 100 age-matched and sex-matched HIV-uninfected control participants recruited from the community or hospital-based internal medicine clinics. All had $\geq 1$ major cardiovascular risk factor (hypertension, diabetes mellitus, smoking or high cholesterol). Next we identified 167 PLWH and 63 uninfected controls older than 40 years of age from Cleveland, USA included in a clinical research registry of studies conducted between 2012 and 2017 by the same principal investigators (GAM and CTL).

\section{Study procedures}

For both cohorts, self-reported demographics, smoking status, and medical history including HIV history and hepatitis $\mathrm{B} / \mathrm{C}$ status were obtained using standardised questionnaires and clinical chart review. Diabetes and hypertension were defined as having a self-reported history of the condition or being on a medication for the condition. Among PLWH, we also recorded current and nadir CD4+ count, time since HIV diagnosis, current ART and total duration of ART. A physical examination was conducted that included height, weight, waist and hip measurements, and blood pressure. All HIV-uninfected subjects were confirmed to be negative with a rapid HIV test. After a 12-hour fast, blood was drawn for clinical labs, including a lipoprotein panel and kidney function. Plasma was stored at $-80^{\circ} \mathrm{C}$ for batched measurements of biomarkers. In Cleveland, HIV-1 RNA level was obtained as part of routine clinical care, but at the time of this study it was not yet available as part of routine care in Uganda.

All subjects underwent non-contrast, ECG-gated cardiac CT for calcium scoring. Scans were performed on a 128-slice multidetector CT scanner in Uganda (Siemens; Munich, Germany) and 64-slice scanner in Cleveland (Siemens). Tube voltage (120-130 kV), gating interval (60\%-70\% of the RR) and slice thickness (2.5-3 $\mathrm{mm}$ ) were similar in Uganda and Cleveland. CAC score was measured offline by local radiologists using similar Siemens Syngo and Leonardo workstations. Calcified lesions were defined as having $\geq 6$ pixels with density $>130$ Hounsfield units, and total CAC score was calculated using the Agatston method. ${ }^{14}$

Biomarkers of inflammation and immune activation were measured in batch from cryopreserved plasma samples collected from Ugandan subjects only. IL-6 was measured by electrochemiluminescence (Meso Scale Diagnostics, Rockville, Maryland, USA). Soluble vascular cell adhesion molecule (R\&D Systems, Minneapolis, Minnesota, USA), soluble tumour necrosis factor $\alpha$ receptor II (R\&D Systems), soluble intercellular adhesion molecule (sICAM; R\&D Systems), sCD14 (R\&D Systems), sCD163 (R\&D Systems) and oxidised low-density lipoprotein (oxLDL; ALPCO, Salem, New Hampshire, USA) were measured by ELISA. Fibrinogen and high-sensitivity $\mathrm{C}$ reactive protein were measured by nephelometry (Siemens).

\section{Statistical analysis}

We first described the demographics and cardiometabolic risk profile of study participants by study group and HIV status. Statistical comparisons between groups (HIV vs control within country; and Uganda vs US) were made using t-tests and Wilcoxon rank-sum tests for continuous variables, and $\chi^{2}$ or Fisher's exact tests for categorical variables as distributionally appropriate. Missingness was $<2 \%$ for all variables except current CD4+ T cell count (missing $\mathrm{n}=31$ of 267 total PLWH; 12\%).

In the primary analysis, we graphically depicted the prevalence of $\mathrm{CAC}=0$, CAC $1-100$ and $\mathrm{CAC}>100$ among the four study subgroups. We used logistic regression to determine correlates of detectable calcified coronary plaque (defined as CAC $>0$; since numbers in the $>100$ category were too small). We examined the effect of country and HIV status in an (1) unadjusted model, 
(2) model adjusted for age and sex, and (3) model fully adjusted for traditional risk factors (HIV status, country, age, sex, diabetes, hypertension, current smoking and total cholesterol). We tested a country $\times$ HIV interaction in the final model. In two sensitivity analyses, we separately excluded from the final model subjects of non-black race and smokers. We repeated the fully adjusted multivariable model among Ugandan subjects only to examine the effects of these traditional risk factors in this population.

Finally, we explored associations between CAC $>0$ and each of the following HIV-specific variables among PLWH in the USA and Uganda: current CD4, nadir CD4, time since HIV diagnosis, ART duration, current abacavir use and current protease inhibitor (PI) use. Similar to the primary analysis, we first built unadjusted models, then models adjusted only for country, and finally demographics adjusted and fully adjusted models. In a similar fashion, we used unadjusted and adjusted logistic regression to explore associations of CAC $>0$ with each of the individual biomarkers measured in Ugandan subjects only. For the inflammation models, we additionally examined whether associations with inflammation biomarkers varied by HIV status by adding an HIV $\times$ biomarker interaction term to the final model. For interactions that were significant at $p \leq 0.1$, we obtained adjusted OR (AOR) for the biomarker effect in separate models of PLWH only versus HIV-uninfected controls only.

A flow chart of the statistical analyses (online supplementary file 1) describes which analyses were performed on the combined data set versus the Uganda data set only. STATA V.14.0 was used for analysis; $p<0.05$ was considered statistically significant.

\section{RESULTS}

The characteristics of the study participants are displayed in table 1 . Ugandans were more likely to be older $(p<0.001)$ and female $(p<0.001)$. Compared with HIV-negative Ugandans, PLWH in Ugandans had lower rates of diabetes $(p<0.001)$, higher rates of hypertension $(\mathrm{p}<0.001)$ and higher high-density lipoprotein cholesterol $(\mathrm{p}=0.025)$. Although PLWH in Uganda had lower body mass index $(\mathrm{p}=0.009)$, their waist to hip ratio was higher $(\mathrm{p}<0.001)$. Compared with PLWH from the USA, PLWH in Uganda had modestly shorter duration of HIV infection $(\mathrm{p}<0.001)$ and modestly lower current CD4 $(\mathrm{p}=0.014)$; however, nearly all participants had a current CD4 count $>200$. ART regimens differed substantially, with more PI and integrase inhibitor use in the USA $(\mathrm{p}<0.001)$.

Figure 1 shows categorical CAC scores $(0,1-100,>100)$ by country and HIV status. CAC scores were substantially lower among Ugandans compared with US subjects $(p<0.001)$; in fact, $91 \%$ of Ugandans had no detectable coronary calcium. Within-country differences in CAC prevalence between PLWH and HIV-negative participants were not statistically significant (12\% vs $6 \%$ for PLWH vs HIV-negative in Uganda, $\mathrm{p}=0.108 ; 47 \%$ vs $49 \%$ in the
USA, $\mathrm{p}=0.797$ ); however, when countries were pooled together, the prevalence of $\mathrm{CAC}>0$ was higher among PLWH (34\% vs 23\%, PLWH vs HIV-negative; $\mathrm{p}=0.012$ ).

In multivariable logistic regression models, Ugandan subjects were significantly less likely to have CAC $>0$ (vs US subjects) after adjustment for age, sex and HIV status (AOR 0.07 (95\% CI 0.04 to 0.14 ), $\mathrm{p}<0.001$ ). The effect remained unchanged after further adjustment for diabetes, hypertension, current smoking and total cholesterol (AOR 0.07 (95\% CI 0.03 to 0.17 ), p<0.001). HIV infection was not associated with $\mathrm{CAC}>0$ in the final model (AOR 1.23 (95\% CI 0.70 to 2.18), $\mathrm{p}=0.470$ ), and there was no statistically significant HIV $\times$ country interaction $(\mathrm{p}=0.20)$. Excluding smokers $(\mathrm{n}=128$ in the USA and $n=8$ Uganda) or those of non-black race $(n=91$ in the USA) did not significantly alter the protective effect of Ugandan nationality (AOR 0.09 (95\% CI 0.03 to 0.23 ) and AOR 0.15 (95\% CI 0.06 to 0.39 ), $\mathrm{p}<0.001$ ), respectively. When the fully adjusted model was restricted to Ugandan subjects only (table 2), current smoking $(\mathrm{p}=0.039)$ and age $(\mathrm{p}=0.036)$ were associated with $\mathrm{CAC}>0$. PLWH in Uganda had a non-significant increased odds of CAC $>0$ (AOR 2.2 (95\% CI 0.74 to 6.4), $\mathrm{p}=0.157$ ).

In models including all PLWH in Uganda and the USA $(n=267)$, the only variable associated with CAC in fully adjusted models was nadir CD4+ count (AOR 0.72 (95\% CI 0.56 to 0.94$)$ per unit increase in natural $\log (\ln )$-transformed nadir $\mathrm{CD} 4+, \mathrm{p}=0.017)$. Current PI use was associated with CAC in unadjusted models (OR 1.95 (95\% CI 1.16 to 3.29), $\mathrm{p}=0.012$ ), but further adjustment for country, age, sex and traditional risk factors attenuated this relationship $(\mathrm{p}>0.1)$.

There were modest differences in biomarkers between HIV-positive and HIV-negative Ugandans. sCD14 was higher in Ugandan PLWH (median (IQR) 1684 (14182061) vs 1383 (1156-1601) pg/mL, PLWH vs HIV-negative; $p<0.001)$, whereas fibrinogen (312 (253-378) vs 366 (296-428) $\mathrm{mg} / \mathrm{dL}$, PLWH vs HIV-negative; $\mathrm{p}=0.001$ ) and oxLDL (49 (31-88) vs 70 (49-161), PLWH vs HIV-negative; $\mathrm{p}<0.001)$ were lower in Ugandan PLWH. All other biomarkers did not vary by HIV status $(p>0.15)$. The association of inflammation and immune activation markers with coronary calcification in Uganda is shown in table 3. In models that adjusted for HIV status, age, sex and traditional risk factors, concentrations of sICAM, sCD163 and oxLDL were all positively associated with $\mathrm{CAC}$ at $\mathrm{p}<0.05$. Furthermore, the strength of the inflammation-CAC relationship appeared to be much stronger among PLWH compared with HIV-negative Ugandans for sCD163 (AOR 25.9 (95\% CI 3.46 to 193.3) for PLWH vs 1.79 (95\% CI 0.21 to 15.31 ) for HIV-negative; $p$ for interaction $=0.03$ ) and statistically borderline stronger for oxLDL (AOR 2.14 (95\% CI 1.17 to 3.94) for PLWH vs 0.87 (95\% CI 0.27 to 2.80 ) for HIV-negative; $p$ for interaction $=0.10)$. 


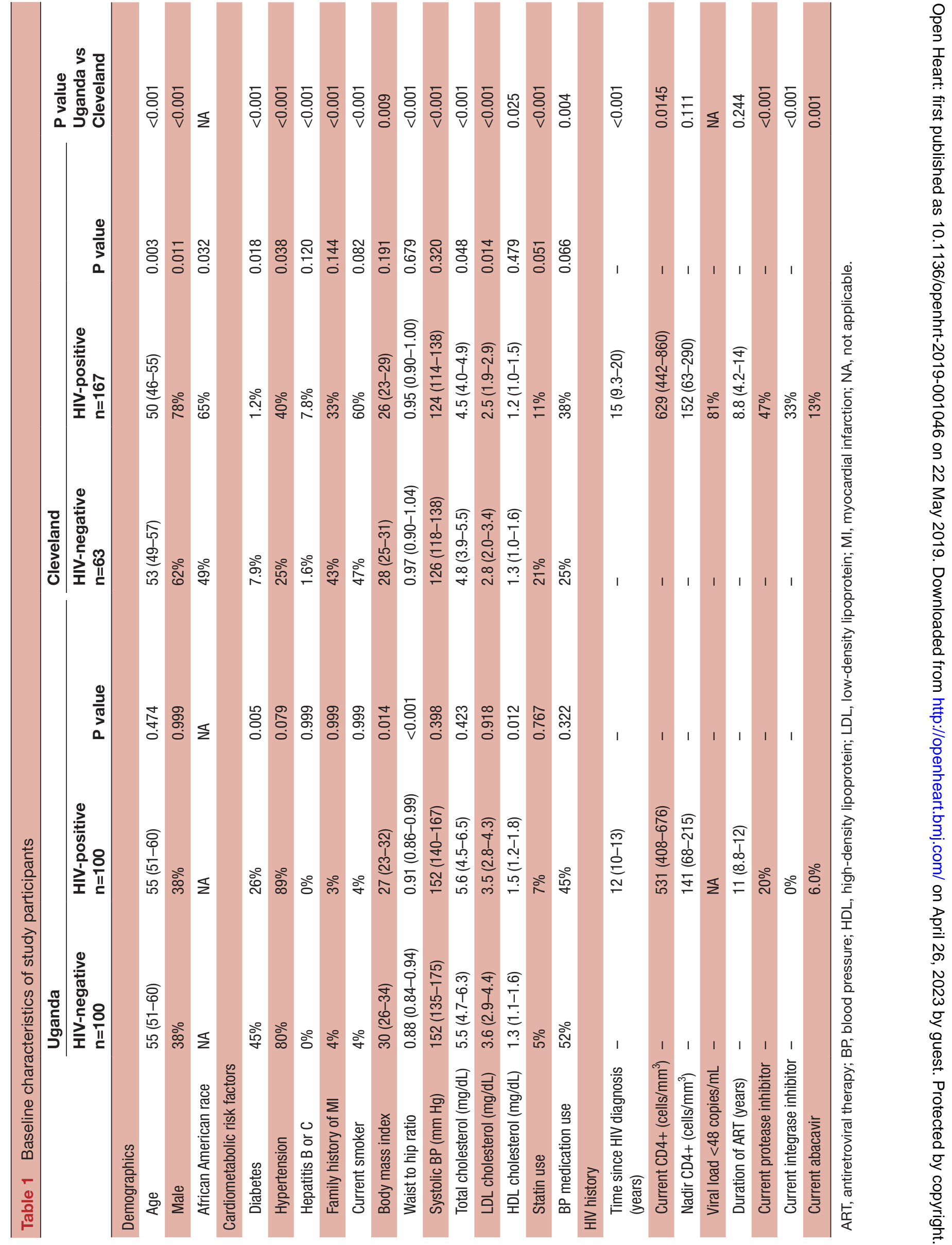




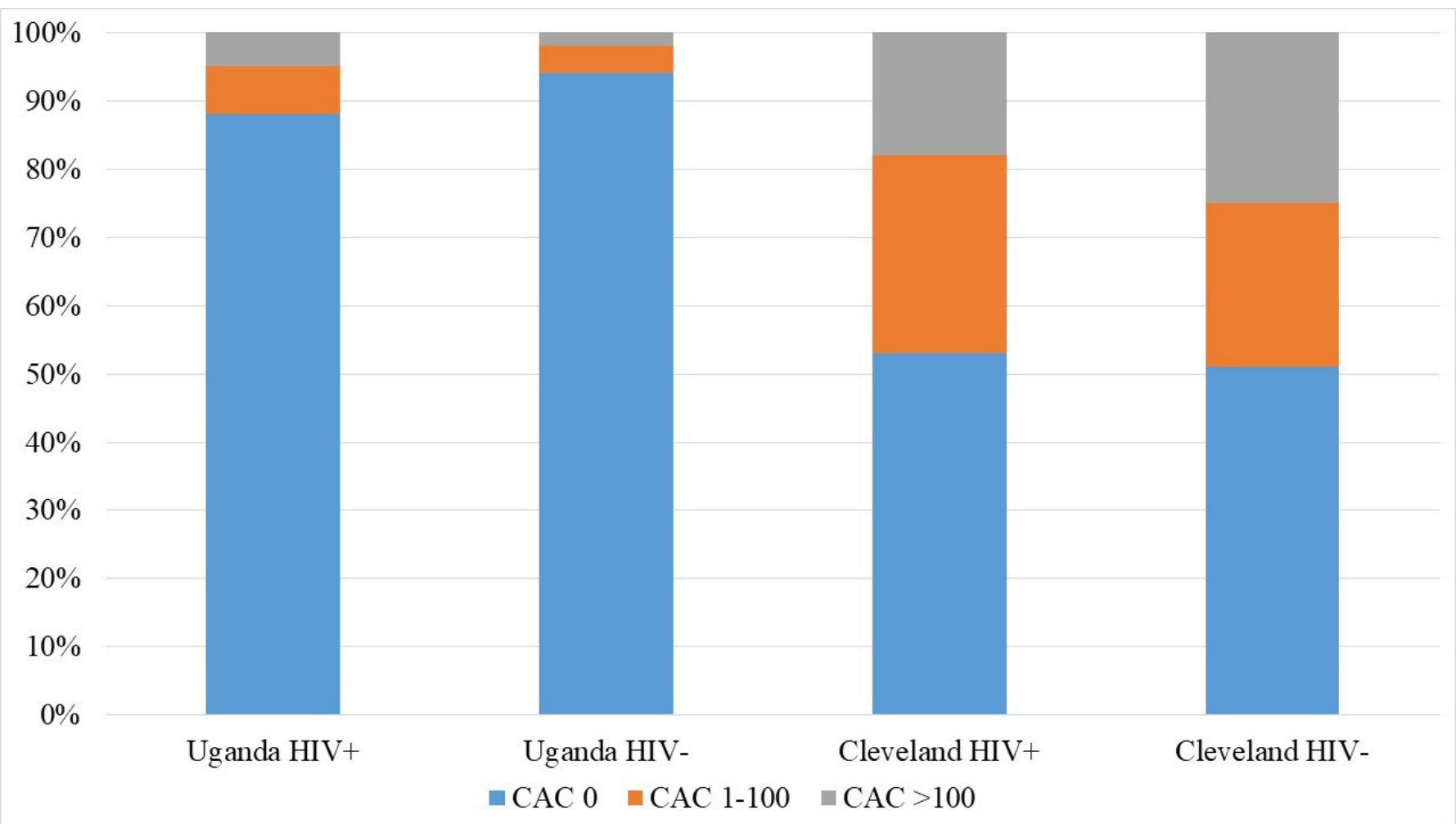

Figure 1 Distribution of coronary artery calcium (CAC) scores among people living with HIV and HIV-uninfected study participants in Uganda and the USA.

\section{DISCUSSION}

The principal finding of our study—that coronary calcium scores are much lower in Ugandans compared with similarly aged persons in the USA, even after adjustment for cardiometabolic risk profile-is novel and challenges current assumptions about rising rates of ischaemic heart disease in SSA. These findings do not appear to be explained by population differences in race or smoking. In addition, we show an inverse association of nadir CD4+ T cell count with CAC among PLWH, and a positive association between biomarkers of inflammation and immune activation and calcified coronary plaque among Ugandans. Finally, the strength of the inflammation-CAC association appears to be strongest for PLWH compared with HIV-uninfected Ugandans. These findings must be interpreted within the context of the limited literature

Table 2 Multivariable model of predictors of detectable coronary calcium $(\mathrm{CAC}>0)$ among Ugandan participants

\begin{tabular}{lcll}
\hline & AOR & $\mathbf{9 5 \%}$ Cl & P value \\
\hline HIV & 2.2 & 0.74 to 6.4 & 0.157 \\
Age (per decade) & 2.3 & 1.1 to 5.1 & 0.036 \\
Male sex & 1.4 & 0.49 to 3.9 & 0.548 \\
Diabetes & 1.3 & 0.41 to 4.4 & 0.629 \\
Hypertension & 5.4 & 0.50 to 59.2 & 0.167 \\
Current smoker & 10.6 & 1.1 to 99.3 & 0.039 \\
\hline Total cholesterol (per $\mathrm{mg} / \mathrm{dL})$ & 1.0 & 0.99 to 1.01 & 0.632 \\
\hline
\end{tabular}

AOR, adjusted OR; CAC, coronary artery calcium. on subclinical coronary disease in SSA and a number of study limitations.

The Global Burden of Disease (GBD) Study 2017 estimates that the age-standardised prevalence of ischaemic heart disease is similar in the USA and Uganda (1776 vs 1293 per 100000 , USA vs Uganda). ${ }^{15}$ In SSA and other areas without primary data on ischaemic heart disease, the GBD Study uses statistical models to impute this disease burden. Moreover, the validity of these models depends on the quality of published data, which are notably lacking in much of SSA. ${ }^{16}$ In the absence of clinical outcomes data, we sought to use coronary calcium scoring as a reliably strong surrogate marker of ischaemic heart disease risk. ${ }^{1718}$ Our findings of dramatically lower rates of CAC in Uganda suggest that rates of clinical ischaemic heart disease might in fact be lower than estimated by GBD; however, further population-based studies and better systems of CVD surveillance are needed to confirm this.

Few published studies have reported on CAC scores in SSA, and none to our knowledge have compared HIV-positive and HIV-uninfected persons. One study of younger, multiethnic South Africans with dialysis-dependent kidney disease ( $\mathrm{n}=40$ blacks and $\mathrm{n}=34$ non-blacks; mean age 42 years, $14 \%$ diabetes) found significantly lower prevalence of $\mathrm{CAC}>0$ among blacks (15\%) compared with non-blacks $(68 \%) \cdot{ }^{19}$ Black AfricanAmericans may have lower coronary calcification in US population-based studies such as the multi-ethnic Study of Atherosclerosis (MESA) and the coronary artery risk 
Table 3 Association of biomarkers of inflammation and immune activation with detectable coronary calcium $(C A C>0)$ among Ugandan participants $(n=200)$

\begin{tabular}{lllll}
\hline & Unadjusted & & \multicolumn{2}{l}{ Multivariable adjusted $^{*}$} \\
\hline IL-6 & OR $(95 \%$ Cl) & P value & AOR & P value \\
\hline sVCAM & $1.20(0.67$ to 2.16$)$ & 0.545 & $1.15(0.61-2.18)$ & 0.666 \\
\hline sTNF-RII & $2.29(0.65$ to 8.10$)$ & 0.199 & $2.21(0.53-9.19)$ & 0.277 \\
hsCRP & $4.81(0.95$ to 24.3$)$ & 0.057 & $4.41(0.71-27.3)$ & 0.110 \\
SICAM & $0.94(0.65$ to 1.37$)$ & 0.748 & $0.94(0.63-1.41)$ & 0.780 \\
\hline Fibrinogen & $3.06(0.97$ to 9.69$)$ & 0.057 & $4.11(1.04-16.2)$ & 0.044 \\
\hline SCD14 & $1.09(0.28$ to 4.34$)$ & 0.899 & $1.13(0.18-7.15)$ & 0.897 \\
\hline SCD163 & $3.49(0.56$ to 21.8$)$ & 0.182 & $1.94(0.26-14.6)$ & 0.520 \\
\hline oxLDL & $3.77(1.27$ to 11.2$)$ & 0.017 & $6.40(1.82-22.6)$ & 0.004 \\
\hline
\end{tabular}

The unadjusted and multivariable adjusted effect size of the association with CAC $>0$ is shown separately for each biomarker. *Adjusted for HIV status, age, sex diabetes, hypertension, current smoking and total cholesterol. All biomarkers were natural log (In)transformed prior to analysis. The effect size is the odds ratio for CAC $>0$ per 1 unit increase in In-transformed biomarker. AOR, adjusted OR; CAC, coronary artery calcium; IL-6, interleukin 6; hsCRP, high-sensitivity C reactive protein; oxLDL, oxidised low-density lipoprotein; sCD14, soluble CD14; sCD163, soluble CD163; sTNF-RII, soluble tumour necrosis factor $\alpha$ receptor II; sVCAM, soluble vascular cell adhesion molecule; siCAM, soluble intercellular adhesion molecule.

development in young adults study (CARDIA). ${ }^{20}$ For example, each SD increase in European ancestry does appear to be associated with a modest $8 \%$ higher CAC prevalence among African-Americans in MESA. ${ }^{21}$ Exclusion of non-black subjects from our final model, however, only modestly attenuated differences in CAC prevalence between the USA and Uganda. It is possible that higher European ancestry among African-Americans compared with Ugandans may be responsible for some degree of residual confounding; however, we feel this is unlikely to account for such dramatic differences in CAC prevalence in our study. Rather, we believe that life-course exposure to risk factors, diet, genetic predisposition and/or physical activity might be responsible for the regional differences we found. Since the process of urbanisation and incorporation of Western diet and lifestyle habits in Uganda is a relatively recent phenomenon, the current older adult Ugandan population is likely to have spent a considerable proportion of their lifetime with a limited risk factor exposure. ${ }^{22}$ These considerations should be examined in future studies of coronary atherosclerosis in SSA.

The presence and extent of CAC are an excellent surrogate marker of the total burden of coronary atherosclerosis and are predictive of future coronary events, across all racial and ethnic groups ${ }^{17}$ and across categories of 10-year Framingham risk. ${ }^{18}$ In particular, a CAC score of 0 is associated with extremely low rates of future ASCVD events among low to intermediate risk asymptomatic patients, and this effect is similar irrespective of race/ ethnicity. ${ }^{23}$ However, future studies using contrast-enhanced CT coronary angiography could provide useful information about non-calcified plaque and high-risk plaque features to further characterise subclinical coronary atherosclerosis in this setting.
Chronic HIV infection, which is known to increase risk for CVD events in the USA and Europe, ${ }^{2}$ has been more strongly associated with non-calcified plaque ${ }^{24}$ and highrisk plaque features ${ }^{25}$ compared with calcified plaque. For example, in the Multicenter AIDS Cohort Study (MACS), HIV-positive status was associated with only modest and statistically borderline higher rates of CAC $>0$ in risk factor-adjusted models (prevalence ratio 1.12 (95\% CI 1.08 to 1.35$)$ ), but the association with non-calcified plaque was somewhat stronger (prevalence ratio 1.25 (95\% CI 1.10 to 1.43$)$ ). In unadjusted analyses, PLWH had higher odds of CAC $>0$ in our study, but similar to MACS this effect was attenuated after adjustment for risk factors. Our findings are consistent with a modest effect of HIV on subclinical coronary atherosclerosis in Uganda; however, we were not adequately powered given the low prevalence of CAC in both groups. Therefore, studies of non-calcified coronary plaque as well as clinical ASCVD event rates are needed. ${ }^{2}$

Among PLWH in our study, lower nadir CD4+ count was associated with higher odds of $\mathrm{CAC}>0$. Prior studies among PLWH suggest that having a history of more severe immunosuppression (ie, low nadir CD4+ T cell count) is associated with more subclinical atherosclerosis, endothelial dysfunction and vascular stiffness. ${ }^{24} 2627$ Lower nadir $\mathrm{CD} 4+\mathrm{T}$ cell count is also associated with higher levels of chronic inflammation and immune activation even after viral suppression on ART. ${ }^{28} 29$

Chronic inflammation is a well-described risk factor for CVD. Among PLWH, markers of inflammation and immune activation have been variably associated with subclinical vascular disease, including CAC, non-calcified plaque and high-risk plaque features. ${ }^{9-12}{ }^{25}$ No studies have previously evaluated these associations among 
HIV and HIV-uninfected persons in low-income and middle-income countries, such as Uganda. Interestingly, we found only modest differences in biomarker concentrations between HIV and HIV-infected subjects in our study. One marker of monocyte activation-sCD14-was higher among PLWH and among women in our cohort, mirroring findings from a south-west Uganda study. ${ }^{30}$ Another marker of monocyte activation-sCD163-was strongly associated with CAC $>0$ in our models after adjustment for traditional risk factors. Interestingly, this association was much stronger among PLWH than among HIV-uninfected Ugandans. Although plasma levels of oxLDL were lower among PLWH in Uganda, the strength of the association with CAC was greater. These findings support immune activation as an important CVD risk marker in SSA, particularly for PLWH. Future studies should further explore how novel drivers of these immune pathways such as chronic infections (eg, cytomegalovirus, malaria, helminths and latent tuberculosis) or exposure to environmental and indoor air pollution may affect cardiovascular risk in this context.

The relatively large sample size for a study from SSA, the inclusion of a US comparison group, and the inclusion of inflammation and immune activation markers are principal strengths of our study. In addition, our study included more women than is typical for studies of PLWH, which increases the generalisability of our findings. Limitations include the cross-sectional design and limited power to detect potentially important associations, including differences between subgroups. Our study subjects were recruited mostly from clinics rather than the community, which may affect the generalisability of our results to the general population. Additionally, differences in baseline risk factor profile between the Uganda and the US subjects allow the possibility of residual confounding.

\section{CONCLUSIONS}

In conclusion, rates of coronary calcification were lower than anticipated in this study of PLWH and HIV-uninfected Ugandans compared with subjects from Cleveland, Ohio, USA. The extent to which HIV infection is associated with subclinical atherosclerosis and/or clinical ASCVD events in SSA requires larger community-based cohort studies. Chronic inflammation and immune activation may be important drivers of subclinical atherosclerosis and clinical ASCVD risk in SSA, particularly for PLWH.

\author{
Author affiliations \\ ${ }^{1}$ Medicine and Pediatrics, University Hospitals Cleveland Medical Center, Cleveland, \\ Ohio, USA \\ ${ }^{2}$ Radiology, St Francis Hospital Nsambya, Kampala, Uganda \\ ${ }^{3}$ Radiology and Medicine, Makerere University College of Health Sciences, Kampala, \\ Uganda \\ ${ }^{4}$ HIV Medicine, Joint Clinical Research Centre, Kampala, Uganda \\ ${ }^{5}$ Cardiology, Uganda Heart Institute, Kampala, Uganda \\ ${ }^{6}$ Radiology, Mackay Memorial Hospital, Taipei, Taiwan \\ ${ }^{7}$ Cardiology, Mackay Memorial Hospital, Taipei, Taiwan
}

${ }^{8}$ Infectious Diseases, Massachusetts General Hospital, Boston, Massachusetts, USA ${ }^{9}$ Medicine, Harvard Medical School, Boston, Massachusetts, USA

${ }^{10}$ Division of Internal Medicine, University Hospital, Sao Paulo, Brazil

${ }^{11}$ Pediatric Infectious Diseases, Case Western Reserve University School of Medicine, Cleveland, Ohio, USA

${ }^{12}$ Cardiology, Case Western Reserve University School of Medicine, Cleveland, Ohio, USA

Contributors BA drafted the manuscript. GE, GM, IS, C-HY, C-LH, MJS, MB and CK contributed to data collection, interpretation of study findings and edited the manuscript for intellectual content. GAM designed the studies, supervised the data collection, interpreted the study findings and edited the manuscript for intellectual content. CTL designed the studies, supervised the data collection, performed the statistical analyses and drafted the manuscript.

Funding This work was supported in part by the National Institutes of Health (K23 HL123341 to CTL; R01 NR012642 to GAM; and R01 HL141053 to MJS).

Competing interests CTL has received a research grant from Gilead Sciences. MB has received a research grant from Sanofi and speaker fee from Boston Scientific. GAM has received grant support and is a consultant for Gilead, ViiV and Merck. No other authors have any disclosures relevant to the content of this manuscript.

Patient consent for publication Not required.

Ethics approval All study procedures conducted in Uganda were reviewed and approved by the Institutional Review Board of University Hospitals Cleveland Medical Center, the Joint Clinical Research Centre (JCRC; Kampala, Uganda) and the Uganda National Council for Science and Technology. The study procedures and research registry in the USA were approved by the IRB of University Hospitals Cleveland Medical Center. All participants in Uganda and Cleveland signed written informed consent.

Provenance and peer review Not commissioned; externally peer reviewed. Data availability statement № additional data are available.

Open access This is an open access article distributed in accordance with the Creative Commons Attribution 4.0 Unported (CC BY 4.0) license, which permits others to copy, redistribute, remix, transform and build upon this work for any purpose, provided the original work is properly cited, a link to the licence is given, and indication of whether changes were made. See: https://creativecommons.org/ licenses/by/4.0/.

\section{REFERENCES}

1. Gersh BJ, Sliwa K, Mayosi BM, et al. Novel therapeutic concepts: the epidemic of cardiovascular disease in the developing world: global implications. Eur Heart J 2010;31:642-8.

2. Shah ASV, Stelzle D, Lee KK, et al. Global burden of atherosclerotic cardiovascular disease in people living with HIV. Circulation 2018;138:1100-12.

3. Hyle EP, Mayosi BM, Middelkoop K, et al. The association between HIV and atherosclerotic cardiovascular disease in sub-Saharan Africa: a systematic review. BMC Public Health 2017;17.

4. Muiru AN, Bibangambah P, Hemphill L, et al. Distribution and performance of cardiovascular risk scores in a mixed population of HIV-infected and community-based HIV-uninfected individuals in Uganda. J Acquir Immune Defic Syndr 2018;78:458-64.

5. Siedner MJ, Kim J-H, Nakku RS, et al. HIV infection and arterial stiffness among older-adults taking antiretroviral therapy in rural Uganda. AIDS 2016;30:667-70.

6. Lazar JM, Wu X, Shi Q, et al. Arterial wave reflection in HIV-infected and HIV-uninfected Rwandan women. AIDS Res Hum Retroviruses 2009;25:877-82.

7. Ngatchou W, Lemogoum D, Ndobo P, et al. Increased burden and severity of metabolic syndrome and arterial stiffness in treatmentnaïve HIV+ patients from Cameroon. Vasc Health Risk Manag 2013;9:509-16.

8. Triant VA, Grinspoon SK. Epidemiology of ischemic heart disease in HIV. Curr Opin HIV AIDS 2017;12:540-7.

9. Vos AG, Hulzebosch A, Grobbee DE, et al. Association between immune markers and surrogate markers of cardiovascular disease in HIV positive patients: a systematic review. PLoS One 2017;12:e0169986.

10. Burdo TH, Lo J, Abbara S, et al. Soluble CD163, a novel marker of activated macrophages, is elevated and associated with noncalcified coronary plaque in HIV-infected patients. J Infect Dis 2011;204:1227-36. 
11. McKibben RA, Margolick JB, Grinspoon S, et al. Elevated levels of monocyte activation markers are associated with subclinical atherosclerosis in men with and those without HIV infection. $J$ Infect Dis 2015;211:1219-28.

12. Longenecker C, Jiang Y, Orringer C, et al. Soluble CD14 is independently associated with coronary calcification and extent of subclinical vascular disease in treated HIV infection AIDS. In Press 2014.

13. Siedner MJ, Kim J-H, Nakku RS, et al. Persistent immune activation and carotid atherosclerosis in HIV-infected Ugandans receiving antiretroviral therapy. J Infect Dis 2016;213:370-8.

14. Agatston AS, Janowitz WR, Hildner FJ, et al. Quantification of coronary artery calcium using ultrafast computed tomography. J Am Coll Cardiol 1990;15:827-32. Jr..

15 Global Burden of Disease Study 2016 (GBD 2016) results2016Seattle, WA Institute forHealth Metrics and Evaluation (IHME), University of Washington

16. DiseaseGBD, Injuryl, PrevalenceCGlobal, et al. Global, regional, and national incidence, prevalence, and years lived with disability for 328 diseases and injuries for 195 countries, 1990-2016: a systematic analysis for the global burden of Disease Study 2016. Lancet 2017;390:1211-59.

17. Detrano R, Guerci AD, Carr JJ, et al. Coronary calcium as a predictor of coronary events in four racial or ethnic groups. $N$ Engl J Med 2008;358:1336-45.

18. Greenland P, LaBree L, Azen SP, et al. Coronary artery calcium score combined with Framingham score for risk prediction in asymptomatic individuals. JAMA 2004;291:210-5

19. Freercks R, Swanepoel C, Carrara H, et al. Vascular calcification in South African dialysis patients: ethnic variation, prevalence, detection and haemodynamic correlates. Nephrology 2012;17:607-15

20. Benjamin EJ, Virani SS, Callaway CW, et al. Heart disease and stroke Statistics-2018 update: a report from the American Heart Association. Circulation 2018;137:e67-492.
21. Wassel CL, Pankow JS, Peralta CA, et al. Genetic ancestry is associated with subclinical cardiovascular disease in AfricanAmericans and Hispanics from the multi-ethnic study of atherosclerosis. Circ Cardiovasc Genet 2009;2:629-36.

22. Kaplan H, Thompson RC, Trumble BC, et al. Coronary atherosclerosis in Indigenous South American Tsimane: a crosssectional cohort study. Lancet 2017;389:1730-9.

23. Greenland P, Bonow RO. How low-risk is a coronary calcium score of zero? The importance of conditional probability. Circulation 2008;117:1627-9.

24. Post WS, Budoff M, Kingsley L, et al. Associations between HIV infection and subclinical coronary atherosclerosis. Ann Intern Med 2014;160:458-67.

25. Zanni MV, Abbara S, Lo J, et al. Increased coronary atherosclerotic plaque vulnerability by coronary computed tomography angiography in HIV-infected men. AIDS 2013;27:1263-72.

26. Ho JE, Deeks SG, Hecht FM, et al. Initiation of antiretroviral therapy at higher nadir CD4+ T-cell counts is associated with reduced arterial stiffness in HIV-infected individuals. AIDS 2010;24:1897-905.

27. Ho JE, Scherzer R, Hecht FM, et al. The association of CD4+ T-cell counts and cardiovascular risk in treated HIV disease. AIDS 2012;26:1115-20.

28. Hunt PW, Martin JN, Sinclair E, et al. T cell activation is associated with lower CD4+ T cell gains in human immunodeficiency virus-infected patients with sustained viral suppression during antiretroviral therapy. J Infect Dis 2003;187:1534-43.

29. Siedner MJ. Start or smart? timing of antiretroviral therapy initiation and cardiovascular risk for people with human immunodeficiency virus infection. Open Forum Infect Dis 2016;3:ofw032.

30. Siedner MJ, Zanni M, Tracy RP, et al. Increased systemic inflammation and gut permeability among women with treated HIV infection in rural Uganda. J Infect Dis 2018;218:922-6. 